\title{
Examination of Countermeasures in a Ward: Novice Nurses' Perception of Issues Relating to Senior Nurses
}

\author{
Kozue Kamijo \\ Nagano College of Nursing, Komagane City, Japan \\ Email: k.kamijo@nagano-nurs.ac.jp
}

How to cite this paper: Kamijo, K. (2020) Examination of Countermeasures in a Ward: Novice Nurses' Perception of Issues Relating to Senior Nurses. Open Journal of Nursing, 10, 209-218.

https://doi.org/10.4236/ojn.2020.103014

Received: February 13, 2020

Accepted: March 9, 2020

Published: March 12, 2020

Copyright (๑) 2020 by author(s) and Scientific Research Publishing Inc. This work is licensed under the Creative Commons Attribution International License (CC BY 4.0).

http://creativecommons.org/licenses/by/4.0/

\section{(c) (i) Open Access}

\begin{abstract}
Purpose: In Japan, demand for nursing is high. However, some novice nurses are unable to adapt to the workplace, and subsequently quit. This study aimed at clarifying relationship issues between novice and senior nurses and examining measures that could be enforced in wards for novice nurses based on the results. Methods: An inductive analysis was conducted on qualitative data from semi-structured interviews with 11 newly hired nurses who had recently graduated from nursing school. The study period was from November 2016 to March 2017. Results: The analysis generated the following three themes related to potential workplace maladaptation: 1) Adaptation to the workplace: difficulty in forming relationships with senior nurses and adapting, 2) Workplace education system: education by preceptors and senior nurses, 3) Professional stance: qualities as a nurse. Conclusion: The results indicated that novice nurses had difficulties in adapting to the workplace as well as its education system in wards. Hence, it was necessary to create habits that would not overly burden others, but instead, spread the rules of the organization, help novice nurses to understand their responsibilities, and assist them in overcoming challenges.
\end{abstract}

\section{Keywords}

Newly Graduated Nurses, Workplace Relationships, New Hire Training, Styling, Workplace Adaptation

\section{Introduction}

Japan's baby boomer generation will enter late adulthood in 2025, and the demand for nursing and care will increase. Novice nurses will therefore, need to 
adapt to the workplace and play an active front line role. However, it has been reported that six months to a year after nurses take up employment, high stress caused by interpersonal relationships influences their desire to leave [1] [2]. Hence, the urgency to: clarify novice nurses' problems at the workplace relating to interpersonal relationships with existing nursing staff; examine the problems in wards accepting novice nurses; and promote workplace adaptation.

In relation to education of novice nurses, since 2010, training programs have been mandatory to ensure medical safety and prevent early turnover of novice nurses [3]. Efforts are being made to train novice nurses that require economic resources and time.

Novice nurses being at the initial stage of working as members of an organization and nursing professionals have the opportunity to socialize and grow in the workplace. Each ward has a workplace culture based on the relationships among the working staff. Since lack of familiarity with workplace culture causes novice nurses to experience a reality shock, maladjustment, and stress in the workplace, it suggests the need for support in workplace relationships [4] [5] [6].

Based on the above, we believe that for novice nurses both, off-the-job and on-the-job training in wards are equally important for promoting their socialization skills. Some measures need to be taken in wards to make novice nurses feel accepted. While previous studies have shown that the relationship between novice and existing nurses is related to factors affecting stress and job separation, no studies have examined specific solutions. To promote socialization of novice nurses, we shall clarify the relationship issues between novice and senior nurses and examine measures that can be implemented in the wards for novice nurses, based on the results.

\section{Objectives}

The objective of this study was to clarify relationship issues between novice and senior nurses and to examine measures that could be implemented in the wards for novice nurses, based on the results.

\section{Study Methods}

\subsection{Study Design}

The study uses a qualitative and inductive design.

\subsection{Definition of Terms}

Novice nurses: nurses employed immediately upon successfully completing a basic nursing education program, who have been working for less than a year, and it is their first working experience.

Workplace culture: working environment based on the interpersonal relationships in the ward to which the novice nurses belong.

Socialization: The process through which individuals acquire the social knowledge and skills needed to fulfill their role in an organization [7]. 


\subsection{Survey Period, Research Participants}

The study period was from November 2016 to March 2017. Participants were nurses who had graduated from a nursing school in March 2016 and started their employment the very next month, viz., April 2016. Since it is generally said that in qualitative research the content is saturated after 10 participants, this study chose 11 .

\subsection{Data Collection}

The research theme and conditions for participating in the research were conveyed verbally and in writing. Thereafter, interviews were conducted with those who returned the consent form. This study conducted the interviews using an interview guide based on the semi-structured interview method. The interviewer asked the participants what they felt about their relationship with senior nurses in the workplace and what they had done to improve (become familiar with) them. One interview of an hour's duration was conducted for each participant.

\subsection{Data Analysis}

The obtained data were analyzed qualitatively and inductively. In the analysis, we read through the transcripts to understand the context of the conversation and then sectioned them so as not to lose the semantic contents of the narratives. We classified the themes of the problems in the relationships between novice and senior nurses, considering the similarities and differences of the sections. Thereafter, we named them considering the semantic contents of the classifications. We examined the data from the viewpoint of novice nurses. We repeated this process to generate categories and subcategories. This process was carried out with another researcher having experience in qualitative research. Furthermore, the study was conducted under the supervision of three researchers specialized in qualitative research.

\section{Ethical Considerations}

We documented the study's outline and purpose, preservation of anonymity, handling of data, and the fact that participation was based on free will. The Integrated Chip (IC) recording was also approved in advance with written and oral explanations. This study was conducted with the approval of the ethical committee of the university A (approval number 2016-16).

\section{Results}

\subsection{Summary of the Study Participants and Interviews}

There were 11 participants ( 2 males and 9 females), all of whom were nurses who graduated from a nursing school in March 2016. The interviews were conducted between 7 and 11 months after their employment. The average interview duration was $47.5 \mathrm{~min}$ (23 - $74 \mathrm{~min}$ ). 


\subsection{Results of the Analysis}

We analyzed the narratives on novice nurses' perceptions of problems with existing nurses and extracted 165 codes. Subsequently, we found 7 subcategories and 3 categories (Table 1 ).

We signify categories in italics, subcategories with " ", codes with ", and narratives with " " and in italics.

\section{1) Adaptation to the workplace}

This category was named Adaptation to the workplace because it consisted of novice nurses who were having trouble building relationships necessary to work within the organization. The subcategories were "Efforts to adapt to the ward", "Difficult to talk", "Mental and physical fatigue". Regarding senior

Table 1. Interview results.

\begin{tabular}{|c|c|c|}
\hline Category & $\begin{array}{l}\text { Subcategory } \\
\text { (Number of codes) }\end{array}$ & Primary code (number of total codes: 165 ) \\
\hline \multirow{14}{*}{$\begin{array}{l}\text { Adaptation } \\
\text { to the } \\
\text { workplace }\end{array}$} & \multirow{4}{*}{$\begin{array}{l}\text { Efforts to adapt } \\
\text { to the ward }(21)\end{array}$} & I had a conversation to get my seniors accepted (7) \\
\hline & & Want to know what factors of novice nurses are preferred by people in the workplace (3) \\
\hline & & Even if it was a scary senior nurse, I understood that I had to report, contact and consult (3) \\
\hline & & I knew I needed a way to talk without being misunderstood (8) \\
\hline & \multirow{5}{*}{ Difficult to talk (22) } & I cannot say or write what is happening (4) \\
\hline & & I am scared to talk to senior nurses (11) \\
\hline & & $\begin{array}{l}\text { It is difficult to know the best time to talk about reports, contacts, or consults, } \\
\text { to senior nurses in a busy atmosphere ( } 3 \text { ) }\end{array}$ \\
\hline & & The quality of care becomes poor as I try to finish early so as not to offend senior nurses (3) \\
\hline & & It is easy to talk to those who explain without being denied (1) \\
\hline & \multirow{5}{*}{$\begin{array}{l}\text { Mental and } \\
\text { physical fatigue (77) }\end{array}$} & Misunderstood with immature attitude (2) \\
\hline & & Don't deny, please care (7) \\
\hline & & Incompatible with the tacit rules of the workplace (37) \\
\hline & & It is painful to be in the workplace (22) \\
\hline & & I am thinking of leaving the job (9) \\
\hline \multirow{6}{*}{$\begin{array}{l}\text { Workplace } \\
\text { education } \\
\text { system }\end{array}$} & \multirow{3}{*}{ Preceptor guidance (10) } & It was different from the expected preceptor (7) \\
\hline & & Preceptor does not receive consultation (2) \\
\hline & & Preceptors can't grasp the meaning of what novice nurses want to convey (1) \\
\hline & \multirow{3}{*}{$\begin{array}{c}\text { Guidance by } \\
\text { senior nurse (15) }\end{array}$} & Inadequate educational plan for new nurses (7) \\
\hline & & I get many different comments from different people (5) \\
\hline & & I am very grateful for the follow-up by my senior nurse (3) \\
\hline \multirow{4}{*}{$\begin{array}{l}\text { Professional } \\
\text { stance }\end{array}$} & \multirow{2}{*}{$\begin{array}{l}\text { Maintaining } \\
\text { motivation (10) }\end{array}$} & Supported by the involvement of people around me, I am barely continue (7) \\
\hline & & I can't lose my motivation to study because there are people who teach me (3) \\
\hline & \multirow{2}{*}{$\begin{array}{l}\text { Nursing as the ideal } \\
\text { that became clear (10) }\end{array}$} & Senior nurse who has the trust of doctors is the ideal nurse image (4) \\
\hline & & I feel a gap in the current situation where I cannot ideally engage with patients (6) \\
\hline
\end{tabular}


nurses, novice nurses said 'I knew I needed a way to talk, without being misunderstood', 'I am scared to talk to senior nurses', 'It is difficult to know the best time for discussing reports and contacts, or consulting senior nurses in a busy atmosphere', 'Incompatible with the tacit rules of the workplace'.

" $P$ m not good at speaking. Perhaps the way I speak causes misunderstandings and it doesn't work well; sometimes I misunderstand them, and vice versa. How to take it? $(B)$ "

"The ones who are scared and awkward to talk to, are those who have mood swings and don't know what to get angry about. If you hesitate to report, she gets more angry because of time being lost $(E)$ "

“When I couldn't attend to a nurse call because of other duties, my senior's stare made me feel that I was being blamed. It's an implicit rule. (E)"

2) Workplace education system

This category was named Workplace education system and consisted of education content for novice nurses. The subcategories were "Preceptor guidance" and "Guidance by senior nurses".

In "Preceptor guidance", the novice nurses said that 'It was different from the expected preceptor' and that 'Preceptors couldn't grasp the meaning of what novice nurses wanted to convey'. In “Guidance by senior nurses”, it was mentioned that senior nurses had 'Inadequate educational plans for novice nurses' and that 'I get many different comments from different people'. In addition, a novice nurse mentioned that 'I am very grateful for the follow-up by my senior nurse'. It was mentioned that novice nurses need the support of senior nurses.

"Since the colleague who quit the job was competent, senior nurses found a sudden increase in their number of patients. Since he/she was unable to handle them, his/her number of patients suddenly decreased while the number of patients for the other colleagues increased. It may have hurt his/her pride as the other colleagues started to have more patients while he/she did not. (C)"

"Senior nurses are separately advised that novice nurses should perform certain tasks, but when they act, as advised, people who don't know the advice may think they' re doing something selfish. (H)"

"Now we have to take care of the patient. I mean, my training is not the first priority and I want senior nurses to take care of patients, without fail $(A)$ "

3) Professional stance

This category was named Professional stance and consisted of content on qualifications as a nurse. The subcategories were "Maintaining motivation" and "Nursing as the ideal that becomes clear".

Novice nurses mentioned the following: 'Supported by the involvement of people around me, I am able to continue', 'Senior nurse who enjoys the trust of doctors is the ideal nurse image', 'I feel a gap in the current situation when I cannot ideally engage with patients'.

"Oh, I think that I don't want to go anymore, but the more I think, the less I want to go, so I decided not to. There was a veteran nurse comforting me when I failed. So, I thought it was OK. (I)" 


\section{Discussion}

Workplace cultures that novice nurses deemed to be problematic regarding their relationships with senior nurses were sorted into the following three categories: Adaptation to the workplace, Workplace education system, and Professional stance. Here, measures for these issues will be examined.

\subsection{Adaptation to the Workplace}

Novice nurses try to socialize in the ward to which they have been assigned and regularly greet their seniors to make "efforts to adapt to the ward". However, because of the senior nurses' attitudes, the novice nurses feel nervous; they gradually feel that 'I am scared to talk to senior nurses'.

The ethical code in the nursing profession, which is a guideline for nurses, mentions that nurses should respect human dignity and rights and always act with warmth and human consideration [8]. This is not just for patients but for everyone with whom they interact. Intimidating, scary, harsh language, and violent and reprimanding attitudes can make other people feel fear, tension, and pain; and can prevent novice nurses from adjusting to the workplace, by making it difficult for them to continue working and causing mental problems [6] [9] [10]. When mental health problems become serious, it may not be possible for nurses to demonstrate their usual abilities; they may need to take leave and may require additional support after returning to the job, adding burdens for both themselves and the ward. Since intimidating and moody attitudes may lead to problems such as maladaptation to the workplace and turnover in novice nurses, they should be addressed, and it is important to create a culture that does not allow such attitudes.

Viewed from the developmental task theory, novice nurses are slightly at a disadvantage because they are new to employment and need to socialize in an unfamiliar organization. In other words, they want to be accepted by their organization and establish a group identity [11]. Novice nurses have a desire to get used to the workplace but at work, sometimes encounter the "inability to do", lose confidence, and react defensively to maintain self-esteem [11].

Suzuki et al. [12] reported that novice nurses cannot be assertive with senior nurses in various situations, such as when something is bothering them or while trying to avoid deterioration of the relationship; hence, they may sometimes hesitate to say what they want to say. Furthermore, it has been reported that nurses in their $20 \mathrm{~s}$ are more sensitive to remarks by senior nurses [10]. Novice nurses in their $20 \mathrm{~s}$ may, therefore, be afraid of trivial remarks by senior nurses. After all, in situations where novice nurses cannot break through an atmosphere that prevents them from talking, it is difficult for them to provide safe care as their reports get delayed. Senior nurses need to understand the current trends of novice nurses and interact with them with an attitude that is not dictated by their moods.

However, novice nurses should also be aware of their responsibilities as nurs- 
ing professionals. If they see that patients are being harmed due to their delayed reports, they should realize that their fear cannot be an excuse. As nursing professionals, they have the responsibility to provide safe medical care. Thus, it may be necessary to teach them such things at an early stage, in addition to their standard nursing education.

\subsection{Workplace Education System}

Regarding the workplace education system, novice nurses noted that 'preceptors could not grasp the meaning of what novice nurses wanted to convey' and felt that preceptors would not be able to capture their feelings. Another finding regarding novice nurses' difficulty in adapting to the workplace was: 'I knew I needed a way to talk without being misunderstood'. Both sides have poor communication skills and experience difficulties in communicating. However, preceptors are young nurses who were newcomers a few years ago, and not all preceptors excel in communication. In addition, we speculate that as the scope and content of a preceptor's work gradually expands, they may not be able to carry out their duties easily during work. Hence, it is conceivable that they may be behaving inadvertently as soon as this happens; and that a way to talk without being misunderstood, is considered to be a necessary skill not only for novice nurses but also for preceptors and senior nurses [13]. In the case of a person-to-person problem, it cannot be solved unilaterally by either side. In some cases, it is necessary to have a dialogue that seeks an understanding by recognizing their differences because they may assume that they know each other but not realize that they fail to understand each other [14].

The desire for novice nurses to be accepted by senior nurses in the ward surfaces in various ways. It is a workplace problem that different senior nurses follow different rules relating to goals, deadlines, and the number of achievements; and this is said to be stressful for novice nurses. Novice nurses were sometimes confused because their education was carried out on a senior nurse's initiative and the contents of the guidance were not well known to the ward staff since what was commonly being done was disseminating and executing the rules decided during the ward/team meeting. To address this, it is necessary to follow appropriate procedural practices, such as getting people to attend the meetings, recording in the minutes the decisions taken, and thoroughly disseminating. All members need to be aware of this.

Novice nurses said that education was prioritized over the patients' safety and care. Nurses are obliged to avoid harm to patients [15]. Organizations must also ensure security. Senior nurses may put pressure on novice nurses due to their enthusiasm for education. However, to avoid escalation, the organization must disseminate instructions that safety should be prioritized.

\subsection{Professional Stance}

Novice nurses were eager to learn while trying to improve themselves, despite facing difficulties. This is maintained by the involvement of others and the 
presence of someone to teach. Novice nurses are adults. Rather than the pedagogy of giving novice nurses the tasks they need to study and learn; it is more appropriate for them to look back on their practice and work independently on learning the tasks they find necessary; termed as andragogy. If the preceptor gives an assignment, the novice nurse must give it priority. Issues that were found to have got postponed were because they had no deadline. In this case, novice nurses may not feel a sense of accomplishment that learning has been useful, which may affect their motivation.

But independent learning, called andragogy is not always possible. A characteristic of andragogy is that learners become dependent during the transitional period. Since novice nurses who have just found employment are in a transitional period, preceptors and senior nurses need to encourage them to learn and think about what and how they can learn [14]. To do this, instead of merely planning the content of a task, the preceptor must be involved each time and there should be deliberations with the novice nurse so that they may be able to identify and recognize the task better.

Three categories emerged as issues faced by novice nurses in relation to senior nurses. Among them is the subcategory 'Incompatible with the tacit rules of the workplace.' What is worrisome from the term 'rules' is that the tasks described so far may be repeated in the ward. Katsuhara et al. [16] pointed out that if similar problems occur repeatedly in the same organization, it may become an ethical rather than an individual problem for the organization because it cannot pursue appropriate measures. They also pointed out that in order to prevent unethical attitudes from getting normalized in an organization, it is necessary to create a culture that prohibits such attitudes. However, Bazerman et al. [13] mentioned that ethical behavior is not always possible, even with the knowledge of ethics. Even if people can think of ethical behaviors in response to being asked what kind of actions are required when they are calm, they may take unethical actions unconsciously when they act compulsively. In a ward that accepts novice nurses, it is necessary to regulate not only patients but also novice nurses and their counterparts so that they do not become unethical, and this must be managed as an organization.

\subsection{Limitations and Future Directions}

The data in this study was obtained from 11 novice nurses. Since we did not conduct interviews with senior nurses, we did not obtain information from both the stakeholders, which is a limitation of this study.

\section{Conclusions}

1) Problems in the relationships between novice and senior nurses can be categorized into: Adaptation to the workplace, Workplace education system, and Professional stance.

2) It was suggested that measures that a ward can take should include creating a workplace habit that does not overly burden others, disseminating the rules of 
the organization, and communicating directly that safety is a priority for the organization. Novice nurses understand their responsibilities and they need to be helped in the challenges they encounter.

\section{Acknowledgements}

We thank all the people who guided and cooperated in the study. We would like to thank Editage (http://www.editage.com/) for English language editing.

\section{Conflicts of Interest}

There is no conflict of interest in this study.

\section{References}

[1] Murata, N., Wakeshima, R. and Nagaie, T. (2015) Study on Changes in Occupational Stress and in the Desire to Quit among New Graduate Nurses. International Nursing Care Research, 14, 47-55.

[2] Yamazumi, Y., Kitagawa, A. and Yasukata, F. (2017) Research on Turnover Intention of Novice Nurses in Their First Six Months of Employment. Kyoritsu Journal of Nursing, 4, 9-17.

[3] Ministry of Health, Labor and Welfare (2010) Policy Report: Training of Novice Nurse Becomes an Obligation from 2010. http://www.mhlw.go.jp/seisaku/2010/01/04.html

[4] Mizuta, M. (2004) Job Adjustment among New Graduate Nurses: The Process of Recovery from Reality Shock and Factors Which Impede Recovery. Journal of Japan Academy of Nursing Science, 27, 91-99.

[5] Uchiyama, K., et al. (2007) A Survey on Stress and Power-Harassment in Novice Nurses. Proceedings of the Japan Nursing Society Mental Health, 38, 173-175.

[6] Yano, I., Idehara, Y. and Aoshiba, E. (2014) Factors Related to Successful Adaptations to the Workplace in Novice Nurses: From the Interview on the Experience after Starting the Career. Bulletin of Nagasaki Prefectural University Department of Nursing and Nutrition, 12, 11-17.

[7] Van Maanen, J. and Schein, E.H. (1979) Toward of Theory of Organizational Socialization. Research in Organizational Behavior, 1, 209-264.

[8] Japan Nursing Association (2003) Ethical Code of Nursing. https://www.nurse.or.jp/nursing/practice/rinri/rinri.html

[9] Kunii, J., et al. (2017) Mental Health Conditions of Nurses in Acute Care Hospitals. Journal of the Japanese Association of Rural Medicine, 66, 27-37. https://doi.org/10.2185/jjrm.66.27

[10] Ohtori, K., et al. (2014) The Research on the Hospital Nurses' Victim Cognition Induced by Senior Nurses' Behaviors. Journal of Health Sciences of Mind and Body, $10,33-42$.

[11] Nishida, Y. (2016) OJT to Support the Growth of Novice Nurses. Igakushoin, Tokyo.

[12] Suzuki, E., et al. (2014) Situations and Reasons Novice Nurses Cannot Be Assertive toward Their Senior Nurses. The Journal of the Japan Academy of Nursing Administration and Policies, 18, 36-46.

[13] Bazerman, M.H. and Tenbrunsel, A.E. (2011/2013) Chiaki Ikemura (trans.). Blind 
Spots of Ethics: When People and Companies Misjudge. NTT Publisher, Tokyo. https://doi.org/10.1515/9781400837991

[14] Udagawa, G. (2019) Organizational Theory Starting with "I Don't Understand" Working with Others. Newspics, Tokyo.

[15] Fry, S.T. and Johnstone, M.J. (2008/2010) Noriko Katada, Aiko Yamamoto (trans.). Ethics in Nursing Practice (3). Japan Nursing Association Publish Inc., Tokyo.

[16] Katsuhara, Y. (2016) Surviving in an Organization. In the Middle of Management and Ethics. Igakushoin, Tokyo. 